\title{
A comparative evaluation of two chromogenic media for surveillance of carbapenem-resistant Enterobacterales with non- carbapenemase-producing or carbapenemase-producing strains other than $b l a_{\mathrm{KPC}}$ : bla $\mathrm{GES}-5$, bla $a_{\mathrm{NDM}-1}$ or bla $a_{\mathrm{VIM}-2}$
}

https://doi.org/10.1515/labmed-2018-0139

Received September 20, 2018; accepted April 15, 2019; previously published online May 15, 2019

\section{Abstract}

Background: Infections caused by carbapenem-resistant Enterobacterales (CREs) are an emerging problem associated with high rates of morbidity and mortality. CREs are divided into two categories (carbapenemase-producing [CP] CRE and non-CP CRE). The most prevalent carbapenemase produced by Enterobacterales is Klebsiella pneumoniae carbapenemase (KPC) in Korea. Rapid identification of CREs is clinically important in infection control precaution. We compared the performance of two chromogenic media (chromID CARBA agar and CHROMagar KPC agar) for non-CP CREs or CP CREs with $b l a_{\text {GES-5 }}$, bla $a_{\mathrm{NDM}-1}$ or $b l a_{\mathrm{VIM}-2}$ in a Korean hospital.

Methods: The study was carried out during a 3-month period from April to June 2017 during the surveillance program for CRE colonization. Antimicrobial susceptibility testing (AST) and polymerase chain reaction (PCR) were performed at the Korean Centers for Disease Control and Prevention.

Results: A total of 45 rectal swabs from 42 hospitalized patients were examined. Sensitivity of both chromID CARBA and CHROMagar KPC were 100\% for CP CREs; and $50 \%$ and $100 \%$ for non-CP CREs, respectively. Specificity of chromID CARBA and CHROMagar KPC were $89.2 \%$ and $70.3 \%$ for CP CRE, respectively; and $76.9 \%$ and $66.7 \%$ for non-CP CRE, respectively.

Conclusions: The CHROMagar KPC is useful to monitor non-CP and CP CREs. The chromID CARBA is efficient

\footnotetext{
*Correspondence: Chang-Hun Park, MD, Department of Laboratory Medicine and Genetics, Samsung Changwon Hospital, Sungkyunkwan University School of Medicine, 158 Palyong-ro, Changwon 51353, Korea; and Department of Laboratory Medicine, Mokpo Hankook Hospital, Mokpo, Korea, Phone: +82-55-233-6097, Fax:+82-55-233-5734, E-Mail: changhun.park@samsung.com. https://orcid.org/0000-0002-0412-3732
}

for rapid detection of $\mathrm{CP}$ CREs requiring high contact precaution.

Keywords: carbapenem-resistant Enterobacterales; carbapenemase; chromogenic agar.

Infections caused by carbapenem-resistant Enterobacterales (CREs) are an emerging problem associated with high rates of morbidity and mortality, particularly among critically ill patients [1]. CREs are usually resistant not only to $\beta$-lactam antimicrobials but also to most other classes of antimicrobial agents [1,2].

Carbapenem resistance is typically caused by two main mechanisms: (1) production of carbapenemases which hydrolyze carbapenem antibiotics (carbapenemaseproducing, CP) and (2) plasmid or chromosome-encoded cephalosporinase and/or extended-spectrum $\beta$-lactamase (ESBL) production associated with outer-membrane impermeability (non-CP) [3]. The CP CREs include those which produce the class A carbapenemases Klebsiella pneumoniae carbapenemase (KPC) and Guiana extended-spectrum $\beta$-lactamase (GES), the class B metallo- $\beta$-lactamases of the integral membrane protein (IMP), New Delhi metallo- $\beta$ lactamase (NDM) and Verona integron-encoded metallo$\beta$-lactamase (VIM) families, and the class D OXA-48-like enzymes [4]. The KPC is the most prevalent carbapenemase produced by Enterobacterales in several countries worldwide [2]. Patients colonized with CRE are considered as the source of transmission in healthcare settings [2].

Rapid identification of patients colonized by CREs is clinically important in infection control precautions. It enables us to choose the antibiotic therapy, especially in intensive care units due to increased mortality rates [5]. Therefore, surveillance cultures are useful in identifying such patients in order to implement infection control measures rapidly [2]. Automated systems are widely used in many clinical laboratories for the identification of bacterial species and antimicrobial susceptibility testing (AST) [6]. However, it is not enough to monitor the CRE by using an automated system. Direct detection of 
carbapenemase genes in clinical specimens can be performed by molecular methods that are not available in every laboratory, as the need for using specific conditions for each target gene makes the tests expensive [7]. Phenotypic approaches using chromogenic agar media have been developed [8]. ChromID ${ }^{\circledR}$ CARBA agar (bioMérieux, Marcy l'Etoile, France) is a chromogenic solid medium supplemented with specific agents that inhibit the growth of Gram-positive and non-carbapenemase producers [8, 9]. CHROMagar ${ }^{\mathrm{TM}}$ KPC agar (Hy-Laboratories, Rehovot, Israel) is a chromogenic solid medium supplemented with agents that inhibit the growth of Gram-positive/Gramnegative carbapenem-sensitive bacteria [2]. There were many reports about the evaluation of these chromogenic agar media mainly for CP CREs with $b l a_{\mathrm{KPC}}$ or $b l a_{\mathrm{VIM}}$ strains (see Supplementary Table 1).

We compared the performance of two chromogenic media for non-CP CREs or CP CREs with carbapenemase other than the $b l a_{\mathrm{KPC}}$ strain in a Korean hospital.

The study was carried out during a 3-month period from April to June 2017 during the surveillance program for CRE colonization. Clinical specimens of rectal swab for CRE surveillance were collected from hospitalized patients. Each clinical specimen was directly inoculated on standard MacConkey agar, chromID CARBA agar and CHROMagar KPC agar plates. The plates were incubated at $37^{\circ} \mathrm{C}$ for $18-24 \mathrm{~h}$. Suspected CRE colonies on the chromID CARBA agar plate were those with blue/green to blue/gray color for the KESC group (Klebsiella, Enterobacter, Serratia and Citrobacter), pink to burgundy for Escherichia coli and brown for Proteeae (Proteus, Providencia and Morganella) [9]. Suspected CRE colonies on CHROMagar KPC agar were those with metallic blue for Klebsiella and Enterobacter species, and dark pink/reddish for E. coli [10]. In the colonies on standard MacConkey agar plates, identification and AST were performed by VITEK II (bioMérieux, Marcy l'Etoile, France) using GN-ID and AST-N224 cards. Specimens identified as CRE by VITEK II were confirmed by disk diffusion or broth microdilution methods for imipenem (IPM), meropenem (MEM) and ertapenem (ERT), and polymerase chain reaction (PCR) was performed to detect carbapenemase genes. The resulting minimum inhibitory concentration (MIC) values were classified into clinical categories susceptible and resistant following the Clinical Laboratory Standards Institute guidelines [11]. Carbapenemase genes $\left(b l a_{\mathrm{KPC}}, b l a_{\mathrm{VIM}}, b l a_{\mathrm{IMP}}, b l a_{\mathrm{NDM}}, b l a_{\mathrm{GES}}\right.$ and $\left.b l a_{\mathrm{OXA}-48}\right)$ were amplified by PCR followed by direct sequencing as in previous reports [12,13]. Six pairs of primers were designed to amplify internal fragments with sizes from 720 to 893 bp (see Supplementary Table 2). Sequence assembly and editing were done by using the Lasergene DNASTAR ${ }^{\text {TM }}$ version 5.06 software (DNASTAR Inc., Madison, WI, USA). Types of carbapenemases were identified by using the National Center for Biotechnology Information (NCBI) Basic Local Alignment Search Tool (BLAST) (http://blast. ncbi.nlm.nih.gov/). If a microorganism had one or more of the carbapenemase genes, the microorganism was defined as CP CRE, otherwise it was defined as non-CP CRE. Disk diffusion, broth microdilution and PCR were performed at the Korean Centers for Disease Control and Prevention. The process of this study is shown in Figure 1. The sensitivity, specificity, positive predictive value (PPV), negative predictive value (NPV) and overall accuracy were calculated for the two chromogenic agar media.

A total of 45 rectal swabs from 42 hospitalized patients were examined (39 samples from 39 patients, two samples each from the other three patients). In 37 specimens, pathogens were identified. Each of the 30 samples had one microorganism: 43.2\% (16/37) yielded E. coli; 18.9\% (7/37), K. pneumoniae; 8.1\% (3/37), Enterobacter cloacae; 5.4\% (2/37), Citrobacter freundii; $2.7 \%$ (1/37), Klebsiella oxytoca; and $2.7 \%$ (1/37), Klebsiella aerogenes. Each of the seven samples had two different microorganisms: $8.1 \%$ (3/37) yielded $K$. pneumoniae with $E$. coli; $2.7 \%$ (1/37), K. pneumoniae with C. freundii; 2.7\% (1/37), K. oxytoca with E. coli; 2.7\% (1/37), E. cloacae with C. freundii; and 2.7\% (1/37), M. morganii with E. coli. Patient No. 5, 19 and 31 were identified with different strains from other samples at 2-week intervals: Patient 5, C. freundii $\rightarrow C$. freundii $+E$. cloacae; Patient 19, E. cloacae $\rightarrow$ K. pneumonia; Patient 31, K. pneumonia $\rightarrow E$.

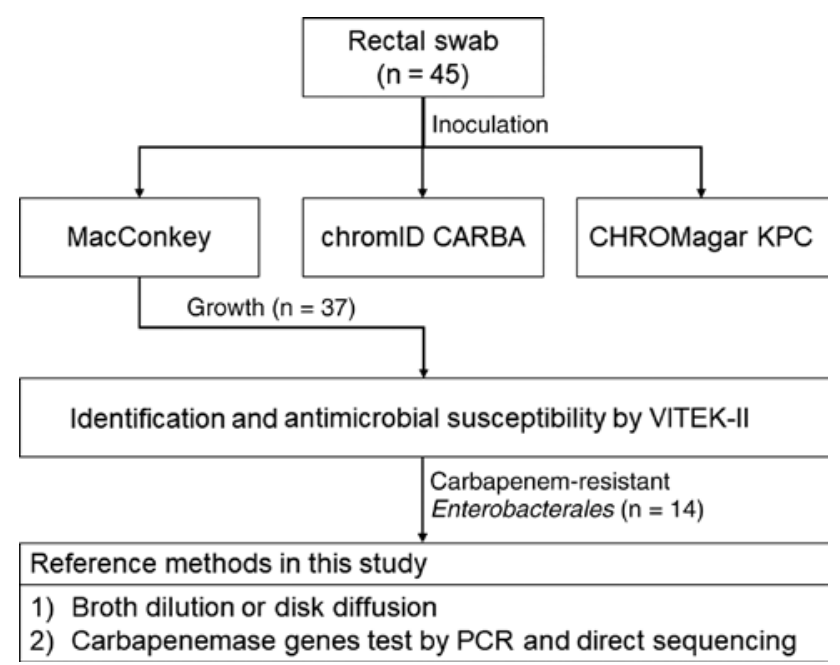

Figure 1: Processing of 45 rectal swabs by the culture on MacConkey agar, chromID CARBA and CHROMagar KPC for the detection of carbapenem resistant Enterobacterales, and final interpretation of the results by antimicrobial susceptibility testing (broth dilution or disk diffusion) and carbapenemase genes test by using PCR and direct sequencing. 
cloacae. Results of the AST showed 14 CREs and 23 carbapenem-susceptible Enterobacterales(CSEs). Of all CREs, 57.1\% (8/14) were CP CREs: $37.5 \%$ (3/8) of CP CREs had $b l a_{\text {GES } 5 \text {; }}$; $37.5 \%$ (3/8), $b l a_{\mathrm{NDM}-1}$; and 25.0\% (2/8), bla VIM -2 . Characteristics of CP CREs identified are summarized in Table 1.

The rates of specimens recovered on chromID CARBA and CHROMagar KPC agar were 32.4\% (12/37) and 51.4\% (19/37), respectively. Sensitivity of both chromID CARBA and CHROMagar KPC agar were similarly high for CP CRE detection in clinical specimens (100\% vs. $100 \%)$ (Table 2). For CP CREs, chromID CARBA had higher specificity as compared to CHROMagar KPC ( $89.2 \%$ vs. $70.3 \%)$. The sensitivity for detecting non-CP CREs on CHROMagar KPC was higher than that on chromID CARBA (100\% vs. 50\%).

CREs are spread worldwide, comprising the source of hospital-acquired infections mainly in long-term care wards. Rapid detection of antibiotic-resistant nosocomial pathogens in the gastrointestinal tracts of patients is considered a necessary step in successful infection control protocols [1, 2]. Early detection of CREs should be applied to prevent spread and reduce infections. Therefore, chromogenic agar is often used for surveillance of CREs. In our study, the sensitivity of chromID CARBA was similar to the sensitivity shown in previous studies on CP CREs with mainly bla $a_{\mathrm{KPC}}$ or $b l a_{\mathrm{VIM}}$ strain $(100 \%$ vs. $92.4-96.5 \%)$ [8, 9]. However, the specificity and PPV of chromID CARBA were lower than those of previous studies $(89.2 \%$ vs. $91.2-96.9 \%$ for specificity; $66.7 \%$ vs. $91.2-93.4 \%$ for PPV) [8, 9]. Previous studies of CHROMagar KPC for the detection of CP CREs with mainly bla $a_{\mathrm{KPC}}$ or bla $a_{\mathrm{VIM}}$ strain showed sensitivity of $84.9 \%-100 \%[2,10,14]$. In the present study, the sensitivity and NPV of CHROMagar KPC was 100\% for all CRE samples regardless of carbapenemase production. Intervention for hand hygiene and contact precaution, which is a basic prophylactic policy for multi-drug resistance, is required for all CREs regardless of carbapenemase production [1]. Considering the aforementioned results, CHROMagar KPC was more suitable for CRE screening than chromID CARBA.

On the other hand, chromID CARBA, compared with CHROMagar KPC, had good specificity as well as sensitivity to the detection of CP CREs (Table 2). As carbapenemase-encoding genes are often located on plasmids, this type of resistance is more likely to disseminate and be capable of causing nosocomial outbreaks [15]. ChromID CARBA is useful in the rapid detection of CP CREs requiring high contact precaution.

The limitation of this study is that the number of samples is small. Due to the characteristics of the hospital

Table 2: Summary of the performances of the two chromogenic methods for the detection of CP CRE vs. non-CP CRE vs. CRE.

\begin{tabular}{lrrrrr}
\hline Methods & Sensitivity & Specificity & PPV & NPV & Accuracy \\
\hline chromID CARBA & & & & & \\
$\quad$ for CP CRE & $100 \%$ & $89.2 \%$ & $66.7 \%$ & $100 \%$ & $91.1 \%$ \\
$\quad$ for non-CP CRE & $50.0 \%$ & $76.9 \%$ & $25.0 \%$ & $90.9 \%$ & $73.3 \%$ \\
$\quad$ for CRE & $78.6 \%$ & $96.8 \%$ & $91.7 \%$ & $90.9 \%$ & $91.1 \%$ \\
CHROMagar KPC & & & & & \\
$\quad$ for CP CRE & $100 \%$ & $70.3 \%$ & $42.1 \%$ & $100 \%$ & $75.6 \%$ \\
$\quad$ for non-CP CRE & $100 \%$ & $66.7 \%$ & $31.6 \%$ & $100 \%$ & $71.1 \%$ \\
for CRE & $100 \%$ & $83.9 \%$ & $73.7 \%$ & $100 \%$ & $88.9 \%$ \\
\hline
\end{tabular}

$\mathrm{CP}$, carbapenemase-producing; CRE, carbapenem-resistant Enterobacterales; NPV, negative predictive value; PPV, positive predictive value.

Table 1: Characteristics of the isolated carbapenemase-producing carbapenem-resistant Enterobacterales.

\begin{tabular}{|c|c|c|c|c|c|c|c|c|}
\hline \multirow[t]{2}{*}{ Patient no. } & \multirow[t]{2}{*}{ Species } & \multirow[t]{2}{*}{ bla type } & \multicolumn{3}{|c|}{ Broth microdilution (MIC, $\mu \mathrm{g} / \mathrm{mL})^{\mathrm{a}}$} & \multicolumn{3}{|c|}{ Disk diffusion (zone diameter, $\mathrm{mm}$ ) } \\
\hline & & & IPM & MEM & ERT & IPM & MEM & ERT \\
\hline $5^{c}$ & Citrobacter freundii & $b l a_{\mathrm{NDM}-1}$ & & & & $18(\mathrm{R})$ & $16(\mathrm{R})$ & $12(\mathrm{R})$ \\
\hline $5^{c}$ & Enterobacter cloacae & $b l a_{\mathrm{VIM}-2}$ & & & & $17(\mathrm{R})$ & $18(\mathrm{R})$ & $16(\mathrm{R})$ \\
\hline 11 & Klebsiella pneumoniae & $b l a_{\text {GES-5 }}$ & $8(\mathrm{R})$ & $8(R)$ & $8(\mathrm{R})$ & & & \\
\hline 23 & Klebsiella pneumoniae & $b l a_{\mathrm{GES}-5}$ & & & & $6(\mathrm{R})$ & $6(\mathrm{R})$ & $6(\mathrm{R})$ \\
\hline 29 & Klebsiella oxytoca & $b l a_{\text {GES-5 }}$ & $1(\mathrm{~S})$ & $\leq 0.5(\mathrm{~S})$ & $1(\mathrm{l})$ & & & \\
\hline 30 & Citrobacter freundii & $b l a_{\mathrm{NDM}-1}$ & & & & $16(\mathrm{R})$ & $16(\mathrm{R})$ & $11(\mathrm{R})$ \\
\hline $31^{c}$ & Klebsiella pneumoniae & $b l a_{\mathrm{NDM}-1}$ & & & & $17(\mathrm{R})$ & $14(\mathrm{R})$ & $9(\mathrm{R})$ \\
\hline $31^{c}$ & Enterobacter cloacae & $b l a_{\mathrm{VIM}-2}$ & & & & $16(\mathrm{R})$ & $17(\mathrm{R})$ & $16(\mathrm{R})$ \\
\hline
\end{tabular}

${ }^{a}$, resistant strain if IPM $\geq 4 \mu \mathrm{g} / \mathrm{mL}, M E M \geq 4 \mu \mathrm{g} / \mathrm{mL}$ and ERT $\geq 2 \mu \mathrm{g} / \mathrm{mL}$; I, intermediate resistant if IPM $2 \mu \mathrm{g} / \mathrm{mL}, \mathrm{MEM} 2 \mu \mathrm{g} / \mathrm{mL}$ and ERT $1 \mu \mathrm{g} /$ $\mathrm{mL}$; S, susceptible isolate if IPM $\leq 1 \mu \mathrm{g} / \mathrm{mL}, M E M \leq 1 \mu \mathrm{g} / \mathrm{mL}$ and ERT $\leq 0.5 \mu \mathrm{g} / \mathrm{mL}$. ${ }^{b} R$, resistant strain if IPM $\leq 19 \mathrm{~mm}, M E M \leq 19 \mathrm{~mm}$ and ERT $\leq 18 \mathrm{~mm}$; I, intermediate resistant if IPM 20-22 mm, MEM 20-22 mm and ERT 19-21 mm; S, susceptible isolate if IPM $\geq 23 \mathrm{~mm}$, MEM $\geq 23 \mathrm{~mm}$ and ERT $\geq 22 \mathrm{~mm}$. 'Different strains identified in the same patients. ERT, ertapenem; IPM, imipenem; MEM, meropenem; MIC, minimum inhibitory concentration. 
where this study was conducted, it was possible to obtain some samples showing rare carbapenemase strains. However, the sufficient number of specimens could not be obtained owing to the short observation period. Nevertheless, it was enough to identify the characteristics of CHROMagar KPC and chromID CARBA agar. This study was conducted only for CP CREs with $b l a_{\text {GES-5 }}, b l a_{\mathrm{NDM}-1}$ and $b l a_{\text {VIM-2. }}$ Therefore, further studies on rare strains such as $b l a_{\mathrm{OXA}}$, bla $a_{\mathrm{GES}}$ and $b l a_{\mathrm{IMP}}$ are needed. Author has complied with the World Medical Association Declaration of Helsinki regarding ethical conduct of research involving human subject and/or animals.

Author contributions: The author has accepted responsibility for the entire content of this submitted manuscript and approved submission.

Research funding: None declared. Employment or leadership: None declared.

Honorarium: None declared.

Competing interests: The funding organization(s) played no role in the study design; in the collection, analysis, and interpretation of data; in the writing of the report; or in the decision to submit the report for publication.

\section{References}

1. Centers for Disease Control and Prevention. Guidance for control of infections with carbapenem-resistant or carbapenemase-producing Enterobacteriaceae in acute care facilities. MMWR Morb Mortal Wkly Rep 2009;58:256-60.

2. Panagea T, Galani I, Souli M, Adamou P, Antoniadou A, Giamarellou H. Evaluation of CHROMagar KPC for the detection of carbapenemase-producing Enterobacteriaceae in rectal surveillance cultures. Int J Antimicrob Agents 2011;37:124-8.

3. Logan LK, Weinstein RA. The epidemiology of carbapenem-resistant Enterobacteriaceae: the impact and evolution of a global menace. J Infect Dis 2017;215:S28-S36.

4. Hornsey M, Phee L, Woodford N, Turton J, Meunier D, Thomas C, et al. Evaluation of three selective chromogenic media, CHROMagar ESBL, CHROMagar CTX-M and CHROMagar KPC, for the detection of Klebsiella pneumoniae producing OXA-48 carbapenemase. J Clin Pathol 2013;66:348-50.
5. Tamma PD, Goodman KE, Harris AD, Tekle T, Roberts A, Taiwo A, et al. Comparing the outcomes of patients with carbapenemaseproducing and non-carbapenemase-producing carbapenemresistant Enterobacteriaceae bacteremia. Clin Infect Dis 2017;64:257-64.

6. He Q, Chen W, Huang L, Lin Q, Zhang J, Liu R, et al. Performance evaluation of three automated identification systems in detecting carbapenem-resistant Enterobacteriaceae. Ann Clin Microbiol Antimicrob 2016;15:40.

7. Schechner V, Straus-Robinson K, Schwartz D, Pfeffer I, Tarabeia J, Moskovich R, et al. Evaluation of PCR-based testing for surveillance of KPC-producing carbapenem-resistant members of the Enterobacteriaceae family. J Clin Microbiol 2009;47:3261-5.

8. Vrioni G, Daniil I, Voulgari E, Ranellou K, Koumaki V, Ghirardi $\mathrm{S}$, et al. Comparative evaluation of a prototype chromogenic medium (ChromID CARBA) for detecting carbapenemase-producing Enterobacteriaceae in surveillance rectal swabs. J Clin Microbiol 2012;50:1841-6.

9. Papadimitriou-Olivgeris M, Bartzavali C, Christofidou M, Bereksi $\mathrm{N}$, Hey J, Zambardi G, et al. Performance of chromID(R) CARBA medium for carbapenemases-producing Enterobacteriaceae detection during rectal screening. Eur J Clin Microbiol Infect Dis 2014;33:35-40.

10. Adler A, Navon-Venezia S, Moran-Gilad J, Marcos E, Schwartz D, Carmeli Y. Laboratory and clinical evaluation of screening agar plates for detection of carbapenem-resistant Enterobacteriaceae from surveillance rectal swabs. J Clin Microbiol 2011;49:2239-42.

11. Clinical and Laboratory Standards Institute. Performance standards for antimicrobial susceptibility testing. Twenty-Seventh Informational Supplement, M100-S27. Wayne, PA: Clinical and Laboratory Standards Institute, 2017.

12. Touati M, Diene SM, Dekhil M, Djahoudi A, Racherache A, Rolain JM. Dissemination of a class I integron carrying VIM-2 carbapenemase in Pseudomonas aeruginosa clinical isolates from a hospital intensive care unit in Annaba, Algeria. Antimicrob Agents Chemother 2013;57:2426-7.

13. Poirel L, Walsh TR, Cuvillier V, Nordmann P. Multiplex PCR for detection of acquired carbapenemase genes. Diagn Microbiol Infect Dis 2011;70:119-23.

14. Samra Z, Bahar J, Madar-Shapiro L, Aziz N, Israel S, Bishara J. Evaluation of CHROMagar KPC for rapid detection of carbapenem-resistant Enterobacteriaceae. J Clin Microbiol 2008;46:3110-1.

15. Carattoli A. Plasmids and the spread of resistance. Int J Med Microbiol 2013;303:298-304.

Supplementary Material: The online version of this article offers supplementary material (https://doi.org/10.1515/labmed-2018-0139). 